\title{
Erratum to: What shapes the intention to study abroad? An experimental approach
}

\author{
Knut Petzold $^{1} \cdot$ Petra $_{\operatorname{Moog}^{2}}$
}

Published online: 6 May 2017

(C) Springer Science+Business Media Dordrecht 2017

\section{Erratum to: High Educ (2017)}

DOI 10.1007/s10734-017-0119-z

The original version of this article unfortunately contained a mistake. Table 5 was incorrect. Coefficients in Table 5 were not aligned to their appropriate labels in the first column. The correct Table 5 is now given below.

The original article was corrected.

The online version of the original article can be found at http://dx.doi.org/10.1007/s10734-017-0119-z

Knut Petzold

knut.petzold@ku.de

1 Department of Sociology, Catholic University of Eichstätt-Ingolstadt, Ostenstraße 26, 85072 Eichstätt, Germany

2 School of Economic Disciplines, University of Siegen, Siegen, Germany 
Table 5 Effects of vignette dimensions and student's characteristics on the intention to study abroad

\begin{tabular}{|c|c|c|c|c|c|c|c|c|c|}
\hline & \multicolumn{9}{|c|}{ Dependent variable: intention to study abroad } \\
\hline & \multicolumn{3}{|c|}{ Model 1} & \multicolumn{3}{|l|}{ Model 2} & \multicolumn{3}{|c|}{ Model $3^{a}$} \\
\hline & $B$ & $S E B$ & Beta & $B$ & $S E B$ & Beta & $B$ & $S E B$ & Beta \\
\hline \multicolumn{10}{|l|}{ Vignette dimensions } \\
\hline Exchange program (ref. no program) & $0.34 * * *$ & $(0.06)$ & 0.09 & & & & $0.34 * * *$ & $(0.06)$ & 0.09 \\
\hline Fin. scholarship (ref. no scholarship) & $0.98 * * *$ & $(0.06)$ & 0.24 & & & & $0.98 * * *$ & $(0.06)$ & 0.24 \\
\hline Exchange in group (ref. alone) & $0.36 * * *$ & $(0.06)$ & 0.09 & & & & $0.36^{* * *}$ & $(0.06)$ & 0.09 \\
\hline \multicolumn{10}{|l|}{ Related language skills } \\
\hline Elementary skills (ref. no skills) & $0.53 * * *$ & $(0.07)$ & 0.12 & & & & $0.53 * * *$ & $(0.07)$ & 0.12 \\
\hline Good skills (ref. no skills) & $1.27 * * *$ & $(0.07)$ & 0.30 & & & & $1.26 * * *$ & $(0.07)$ & 0.30 \\
\hline \multicolumn{10}{|c|}{ Reputation host university } \\
\hline Equal reputation (ref. poorer rep.) & $0.26 * * *$ & $(0.07)$ & 0.06 & & & & $0.27 * * *$ & $(0.07)$ & 0.06 \\
\hline Better reputation (ref. poorer rep.) & $1.29 * * *$ & $(0.07)$ & 0.30 & & & & $1.28 * * *$ & $(0.07)$ & 0.30 \\
\hline \multicolumn{10}{|l|}{ Host country preference } \\
\hline Less desired (ref. not desired) & $0.58 * * *$ & $(0.07)$ & 0.13 & & & & $0.57 * * *$ & $(0.07)$ & 0.13 \\
\hline Strongly desired (ref. not desired) & $0.75 * * *$ & $(0.07)$ & 0.18 & & & & $0.75 * * *$ & $(0.07)$ & 0.18 \\
\hline \multicolumn{10}{|l|}{ Family's/friends' expectations } \\
\hline Show no expect. (ref. exp. stay) & $0.30 * * *$ & $(0.07)$ & 0.07 & & & & $0.30 * * *$ & $(0.07)$ & 0.07 \\
\hline Expect study abroad (ref. exp. stay) & $0.41 * * *$ & $(0.07)$ & 0.10 & & & & $0.41 * * *$ & $(0.07)$ & 0.10 \\
\hline \multicolumn{10}{|c|}{ Student's characteristics } \\
\hline Self-assessed productivity & & & & 0.07 & $(0.05)$ & 0.04 & 0.06 & $(0.05)$ & 0.03 \\
\hline Believe in personality development & & & & $0.31 * * *$ & $(0.06)$ & 0.18 & $0.31 * * *$ & $(0.06)$ & 0.18 \\
\hline Believe in signaling productivity & & & & -0.04 & $(0.05)$ & -0.03 & -0.08 & $(0.05)$ & -0.06 \\
\hline Personal norm to study abroad & & & & $0.23 * * *$ & $(0.04)$ & 0.20 & $0.20 * * *$ & $(0.04)$ & 0.17 \\
\hline $\begin{array}{l}\text { Constant } \\
\text { Variance }\end{array}$ & $1.22 * * *$ & $(1.13)$ & & $1.34 * * *$ & $(0.33)$ & & -0.35 & $(1.20)$ & \\
\hline$\sigma_{v}$ & $1.02 * * *$ & $(0.05)$ & & $0.74 * * *$ & $(0.05$ & & $0.78 * * *$ & $(0.04)$ & \\
\hline$\sigma_{\epsilon}$ & 1.40 & $(0.02)$ & & 1.80 & $(0.03)$ & & 1.40 & $(0.02)$ & \\
\hline Log likelihood & & -4565.66 & & & -5012.82 & & & -4503.90 & \\
\hline LR- $x^{2}$ & & $997.56 * * *$ & & & $103.24 * * *$ & & & $1121.08 * * *$ & \\
\hline $\mathrm{N}_{\text {vignettes }}$ & & 2456 & & & 2456 & & & 2456 & \\
\hline $\mathrm{N}_{\text {respondents }}$ & & 307 & & & 307 & & & 307 & \\
\hline
\end{tabular}

Random intercept models; maximum likelihood estimation; non-standardized coefficients (B) and standardized coefficients (Beta); standard errors are in parentheses

$* p<0.05 ; * * p<0.01 ; * * * p<0.001$

${ }^{\text {a } C o v a r i a t e s: ~ a g e ~, s e x, ~ r e l a t i o n s h i p ~}\left(-0.25^{*}\right)$, study abroad experience, parents worked abroad, filed of studies (economics, engineering), and origin region (Africa, Western Europe, Eastern Europe and Russia, Southern Europe, China, USA) 